La revue La revue pour l'histoire du CNRS

POUR LHISTORE DU CNRS $\quad 16 \mid 2007$

L'expertise scientifique

Le Fonds national de la recherche scientifique en Belgique

Une expérience originale de l'organisation de la science

Kenneth Bertrams

C OpenEdition

Journals

Édition électronique

URL : https://journals.openedition.org/histoire-cnrs/1590

DOI : 10.4000/histoire-cnrs. 1590

ISSN : 1955-2408

Éditeur

CNRS Éditions

Édition imprimée

Date de publication : 3 avril 2007

ISBN : 978-2-271-06453-0

ISSN : 1298-9800

Référence électronique

Kenneth Bertrams, "Le Fonds national de la recherche scientifique en Belgique », La revue pour I'histoire du CNRS [En ligne], 16 | 2007, mis en ligne le 26 mars 2009, consulté le 20 mai 2021. URL :

http://journals.openedition.org/histoire-cnrs/1590; DOI : https://doi.org/10.4000/histoire-cnrs.1590

Ce document a été généré automatiquement le 20 mai 2021.

Comité pour l'histoire du CNRS 


\section{Le Fonds national de la recherche scientifique en Belgique}

Une expérience originale de l'organisation de la science

\section{Kenneth Bertrams}

1 Si l'on remonte aux origines de cette institution, on ne peut être que frappé par le caractère instable et incertain $d u$ contexte dans lequel il tire ses racines. Cette instabilité est due à la prégnance des clivages traditionnels d'ordre culturel et économique (particulièrement marqués au sein du paysage universitaire), à la position subordonnée qu'ont longtemps occupée la science et la recherche scientifique dans les milieux privés et publics belges et, enfin, à l'occupation du territoire durant la première guerre mondiale.

2 Ce dernier facteur, davantage conjoncturel, est pourtant crucial. On perçoit mieux aujourd'hui l'importance $\mathrm{du}$ contexte militaire dans la reconfiguration des communautés scientifiques sous la bannière des nations. Pour certains savants, la rupture de l'ordre international provoquée par la mobilisation scientifique fournissait l'occasion de décloisonner leurs pratiques en les alignant sur des objectifs militaroindustriels précis, un horizon radicalement éloigné des valeurs universalistes traditionnellement associées à l'éthos ${ }^{1}$ des milieux scientifiques ${ }^{2}$. Le modèle de référence incarné jusque-là par la science allemande n'a fait qu'exacerber ce recentrage politique fondé, en une alchimie inédite, sur la culture du ressentiment et celle de l'application des découvertes scientifiques.

3 Ces deux tendances sont spécialement décelables dans les prises de position des délégués français et belges lors des conférences préliminaires qui ont amorcé, au lendemain de la guerre, la création d'un Conseil international de la recherche ${ }^{3}$. Censé combler le vide associatif depuis la dissolution des conventions scientifiques internationales, le Conseil international consistait en une fédération des différents conseils nationaux constitués pour la plupart durant le conflit. Du côté belge, où une telle structure faisait défaut du fait de l'occupation, la pression s'exerça auprès des pouvoirs publics pour mettre sur pied un Conseil national belge de recherches, à l'image du National Research Council fondé en 1916 aux États-Unis sous l'impulsion du 
physicien George Hale. Mais le gouvernement, acculé par le fardeau des enjeux immédiats et quelque peu réticent à l'idée de suivre le projet des académiciens, ne jugea pas utile de donner suite.

Cette décision eut des retombées déterminantes; elle allait priver les pouvoirs publics belges du pouvoir d'initiative dans le processus d'organisation des activités scientifiques. Contrairement au cas français, où l'administration de la Guerre et les relais publics en général ont joué un rôle décisif dans le démarrage des premières formes de coordination scientifique - pensons à la genèse de l'office national des recherches scientifiques, industrielles et des inventions dirigé par Jules-Louis Breton ${ }^{4}$ -, le cas belge se singularise par une sous-représentation des élites politiques et parlementaires au détriment d'un groupe d'acteurs issus pour la plupart des milieux privés et investis cependant d'un idéal patriotique de modernisation des structures économiques.

L'origine du système « francquiste »

5 On retrouve, au centre de ce groupe, un personnage pour le moins atypique et controversé, Émile Francqui. Nommé à la direction du premier holding financier du pays, la Société générale de Belgique, il décide de s'impliquer dans l'organisation du Comité national de secours et d'alimentation (CNSA), créé pour assurer le ravitaillement de la population civile. Du fait de l'enlisement du conflit, le Comité sera perçu comme le « second gouvernement » de la Belgique en guerre ${ }^{5}$. Francqui occupe, à ce titre, une position de choix dans l'establishment belge. Mobilisant ses réseaux privés et professionnels, il parvient à doter le CNSA d'un budget important, auquel s'associe bientôt une nouvelle structure américaine, le Committee for Relief in Belgium (CRB). Son initiateur n'est autre que Herbert Hoover, futur président des États-Unis, que Francqui avait croisé en Chine au début du siècle lors de négociations relatives à la construction et la concession de plusieurs lignes de chemin de fer. La coopération perdurera entre les deux institutions jusqu'à la signature de l'Armistice, non sans certaines difficultés liées principalement aux contraintes de la guerre et à la personnalité de chacun de leurs « patrons ${ }^{6}$.

Or, dès 1915, Francqui songeait à l'après-guerre et à la manière dont on allait pouvoir affecter, en Belgique, le reliquat financier présumé du CNSA. Il convoqua en toute discrétion une série de responsables universitaires et leur fit part de la somme impressionnante pour l'époque - de 50 millions de francs belges. Pourquoi avoir convié à ce stade les responsables des universités ? Premièrement, il faut savoir que, pour des motifs variables, les quatre universités du pays n'ont pas ouvert leur porte à la rentrée académique de 1914. Aussi bien à Liège et Gand (universités d'État) qu'à Bruxelles et Louvain (universités "libres »), les autorités académiques ${ }^{7}$ étaient relativement disponibles. Par ailleurs, les destructions commises par les Allemands lors de l'invasion du territoire ont contribué à accroître le capital de sympathie de l'opinion publique visà-vis des universités et, en particulier, à l'égard de l'Université catholique de Louvain dont la bibliothèque fut ravagée par un incendie. Enfin, sur un plan plus personnel, Francqui côtoyait plusieurs universitaires de grand calibre (dont Félicien Cattier, docteur en droit et ancien professeur à l'Université libre de Bruxelles) et était sensible au relatif cloisonnement social et scientifique des universités belges. Ces raisons cumulées font que Francqui connaissait parfaitement la situation précaire de l'enseignement supérieur en Belgique et qu'il souhaitait y remédier d'une façon ou d'une autre. 
$7 \quad$ Afin de ne pas heurter les sensibilités en présence, il importait de partir des structures préexistantes. Dès lors, parmi les différents projets esquissés au fil des sept réunions qui eurent lieu d'avril à juin 1916, c'est celui d'une Fondation universitaire qui rencontra l'adhésion du plus grand nombre. L'idée sous-jacente de cette institution à créer au sortir de la guerre était d'octroyer des subsides d'étude et de recherche à des jeunes gens méritants. Mais la Fondation était aussi pensée comme un espace de rencontre et de sociabilité des professeurs d'université belges et étrangers. De ce point de vue, elle procédait d'un subtil équilibre entre conceptions traditionnelles et modernes de l'enseignement et de la recherche universitaires.

8 Le plus délicat, toutefois, avait trait au volet financier - comment s'assurer, une fois la guerre terminée, de l'intangibilité des sommes préservées, notamment à l'égard du gouvernement en exil ? Francqui allait y trouver l'occasion, dès l'Armistice venue, de déployer son habileté de négociateur. En toute discrétion, il s'arrangea avec Hoover pour faire passer pour des largesses américaines - la CRB en l'occurrence - les excédents budgétaires émanant du seul CNSA, ce qui rendait caduque toute tentative de "nationalisation» du projet. Parallèlement, le tandem Francqui-Hoover décida d'allouer directement deux tiers des sommes épargnées (près de 100 millions de francs belges) aux établissements d'enseignement supérieur selon une grille de répartition favorisant les quatre universités. Complètement floués par la tactique, les responsables politiques s'empressèrent d'avaliser le projet de loi qui en résultait. D'autres donations privées vinrent se greffer sur l'initiative et contribuer à la légitimer. Il ne fait guère de doute que la manœuvre dont elle fut issue, élaborée aux frontières de la légalité, accrut le potentiel scientifique des universités belges en un temps record.

L'installation du FNRS

9 Par convention plus que par conviction, l'historiographie tient le discours du roi Albert, prononcé le 1er octobre 1927 à l'occasion du $110^{e}$ anniversaire des Établissements industriels Cockerill à Seraing, pour la cause immédiate du démarrage du FNRS. Et il est vrai que, contrairement à d'autres déclarations solennelles, le "discours de Seraing " frappe par son élan quasi programmatique et son ton mobilisateur. La figure morale $\mathrm{du}$ monarque, sa position située naturellement au-delà des clivages partisans l'imposent comme le relais privilégié d'une cause patriotique indiscutable depuis la fin de la guerre: l'essor des laboratoires scientifiques en liaison avec le développement économique du pays.

La conception retenue dans le discours est celle d'une "science utile, patriotique, sociale et pacifique ${ }^{8} "$; elle est explicitement destinée aux bailleurs de fonds potentiels - industriels, commerçants et financiers. L'orientation qui s'en dégage reflète les positions défendues par Armand Solvay ${ }^{9}$, Dannie Heineman ${ }^{10}$ et Émile Francqui que le roi avait pris soin de consulter au préalable. Si l'on ajoute à cela le fait que la conjoncture politique et économique s'était provisoirement stabilisée, on peut dire que le contexte était particulièrement indiqué pour effectuer une transition qualitative dans le domaine de la recherche scientifique.

11 Sans réelle surprise, Francqui réclame et obtient la présidence du comité de propagande chargé de récolter les fonds d'installation du FNRS. Les grandes entreprises industrielles et bancaires répondent à l'appel. Trois mois après le début de l'opération, la barre des 100 millions de francs belges est dépassée. Bien qu'on puisse parler d'engouement, le mythe d'un enthousiasme «national» véhiculé par la presse de 
l'époque est contredit par les chiffres : $11,7 \%$ des donateurs (institutionnels compris) souscrivent pour $92,2 \%$ du montant total.

Quoi qu'il en soit, le FNRS est constitué dès le mois de février 1928, à la veille du centenaire de l'indépendance de la Belgique. Organisé en vingt-quatre commissions scientifiques, chargées de l'évaluation et de la recommandation des dossiers auprès du conseil d'administration, le Fonds devient rapidement une courroie de transmission privilégiée de la recherche universitaire et constitue, à ce titre, un maillon essentiel de la chaîne institutionnelle esquissée par Francqui douze ans plus tôt ${ }^{11}$. À vrai dire, celuici s'appuyait sur un antécédent étranger, le National Research Fund américain, dont le concepteur était sa vieille connaissance, Herbert Hoover, alors secrétaire d'État au Commerce.

13 En quelques années à peine, d'une guerre à l'autre, le FNRS est passé du statut d'initiative louable à celui d'une institution indispensable pour le développement de la recherche scientifique nationale. Cette métamorphose s'est opérée sur fond de légitimation du système "francquiste" dans son ensemble. Ainsi, quand l'État manifeste subitement son intérêt pour la recherche scientifique, au lendemain de la seconde guerre mondiale, le terrain est déjà balisé. Les pouvoirs publics n'ont plus qu'à avaliser de fait sinon de droit la structure qu'Émile Francqui a conçue à leur insu et, d'une certaine manière, échafaudée contre eux.

Ce n'est pas un hasard si le successeur et «fils spirituel » de Francqui, Jean Willems, directeur du FNRS et des organismes associés pendant près de quarante ans (1930-1970), se définissait fièrement comme un «agent libre » de toute ingérence de l'État ${ }^{12}$.

\section{BIBLIOGRAPHIE}

Universités et entreprises. Milieux académiques et industriels en Belgique, 1880-1970. Kenneth Bertrams, Éditions Le Cri, Bruxelles, 2006.

Histoire des sciences en Belgique : 1815-2000. Robert Halleux, Jan Vandersmissen, Andrée DespyMeyer et Geert Vanpaemel (dir.), Bruxelles, La Renaissance du Livre-Dexia Éditions, 2001, 2 tomes.

\section{NOTES}

1.L'éthos représente le style que doit prendre un orateur pour capter l'attention et gagner la confiance de l'auditoire, pour se rendre crédible et sympathique. Il s'adresse à l'imagination de l'interlocuteur.

2.Voir les contributions reprises dans Le sabre et l'éprouvette. L'invention d'une science de guerre (14-18 Today, Aujourd'hui, Heute, n6), Paris, Noésis, 2003.

3.La première Conférence des Académies des Sciences interalliées eut lieu à Londres du 9 au 11 octobre 1918 ; la seconde à Paris du 26 au 29 novembre 1918. Les discussions 
débouchèrent sur la création d'un Conseil international de la recherche dont l'Assemblée constitutive se tint au Palais des Académies de Bruxelles du 18 au 28 juillet 1919.

4.Yves Roussel, «L'histoire d'une politique des inventions, 1887-1918 ", Cahiers pour l'histoire du CNRS, 1939-1989, 1989-3, pp. 19-57.

5.Au lendemain de l'invasion allemande, le gouvernement belge s'exilera à Anvers puis à Sainte-Adresse, dans les faubourgs du Havre. La création du CNSA revient principalement à l'initiative de l'industriel Ernest Solvay et de Dannie Heineman, financier et ingénieur de nationalité américaine. Voir Liane Ranieri, Dannie Heineman. Un destin singulier (1872-1962), Bruxelles, Racine, 2005, pp. 81-84.

6.Voir Liane Ranieri, Émile Francqui ou l'intelligence créatrice, Paris-Gembloux, Duculot, 1985, pp. 115-181.

7.L'arrestation et la déportation, en mars 1916, de Paul Frédéricq et d'Henri Pirenne, tous deux professeurs à l'université de Gand et farouches opposants à la politique de flamandisation de cette institution soutenue par les Allemands, marquent un tournant dans l'attitude de l'occupant à l'égard des responsables universitaires.

8.Robert Halleux et Geneviève Xhayet, « La marche des idées », dans Robert Halleux, Jan Vandersmissen, Andrée Despy-Meyer et Geert Vanpaemel (dir.), Histoire des sciences en Belgique : 1815-2000, Bruxelles, La Renaissance du Livre-Dexia Éditions, 2001, t. 2, p. 15. 9.Fils aîné d'Ernest Solvay, Armand Solvay (1865-1930) reprend la direction de l'entreprise familiale. Vice-président du Comité central industriel, la principale fédération patronale belge, et membre de la Société chimique de Belgique, il s'intéresse de près au rapprochement des milieux académiques et industriels. À cet égard, il sollicite en mai 1927 la rédaction d'une « Note sur l'action du Comité national de chimie dans la question de la collaboration de la science et de l'industrie » dont l'influence sera grande au moment de la création du FNRS.

10.D'origine américaine et ingénieur de formation, Dannie Heineman (1872-1962) est nommé en 1905 administrateur-délégué de la Société financière de transports et d'entreprises industrielles (la Sofina), un holding très actif dans les secteurs de la traction et de l'électricité. Il en poursuit l'expansion en Europe et en Amérique latine, notamment sur le plan des contrôles et des expertises, jusqu'à son retrait des affaires en 1955. Européen convaincu, mécène et bibliophile, Heineman aura les faveurs du roi Albert qui appréciera, à l'occasion de la création du FNRS, « ses conceptions et ses vues transcendantes sur l'avenir du monde » (cité dans Liane Ranieri, Dannie Heineman, op. cit., p. 372).

11.Le FNRS aura ses quartiers rue d'Egmont à Bruxelles, tout comme la plupart des «fondations scientifiques » liées à Francqui : la Fondation universitaire, le Fonds de la recherche scientifique médicale, la fondation Francqui et l'Institut interuniversitaire des sciences nucléaires.

12.Voir le savoureux portrait qu'en dresse la sociologue américaine Renée C. Fox, Le Château des Belges. Un peuple se retrouve, Paris-Gembloux, Duculot, 1997, pp. 50-62, ici p. 57. 


\section{RÉSUMÉS}

Créé en 1928, le Fonds national de la recherche scientifique (FNRS) est bien le grand frère de son homologue français. Cependant, hormis la parenté chronologique, géographique et lexicographique qui relie les deux institutions, l'inventaire des différences dépasse de loin celui des points communs. Sur le triple plan des conditions d'institutionnalisation, de la nature de l'organisation et $\mathrm{du}$ fonctionnement et, enfin, de l'orientation scientifique proprement dite, le Fonds belge se distingue nettement des organismes similaires de coordination des activités scientifiques créés à l'étranger, plus ou moins simultanément. Il est nécessaire de décrypter les différents sédiments de ce moment fondateur.

Founded in 1928, the National Fund for Scientific Research in Belgium is the older brother of the French CNRS. However, beyond this chronological, geographical and linguistic parenthood, the differences between these two institutions largely outweigh their common points. The FNRS distinguishes itself from the scientific centres created about the same time in other countries on three aspects: the conditions of the institutionalisation, the nature of the organisation and, last but not least, the scientific orientations of the research programme. Hence, it is necessary to examine the basis of this founding moment.

\section{INDEX}

Mots-clés : Fonds national de la recherche scientifique en Belgique, FNRS, Belgique

\section{AUTEUR}

\section{KENNETH BERTRAMS}

Kenneth Bertrams est docteur en histoire et licencié en philosophie de l'Université libre de Bruxelles. Il est chargé de recherches au Fonds national de la recherche scientifique. 